\title{
Identification of Mucorales by Matrix-Assisted Laser Desorption Ionization Time-of-Flight Mass Spectrometry
}

\author{
Patrick Schwarz ${ }^{1,2, *(\mathbb{D})}$, Houssem Guedouar ${ }^{3}$, Farah Laouiti ${ }^{3}$, Frédéric Grenouillet ${ }^{4}(\mathbb{D})$ and \\ Eric Dannaoui ${ }^{3,5}$ (D) \\ 1 Department of Internal Medicine, Respiratory and Critical Care Medicine, University Hospital Marburg, \\ D-35043 Marburg, Germany \\ 2 Center for Invasive Mycoses and Antifungals, Philipps University Marburg, D-35037 Marburg, Germany \\ 3 Université Paris Descartes, Faculté de Médecine, AP-HP, Hôpital Européen Georges Pompidou, \\ Unité de Parasitologie-Mycologie, F-75015 Paris, France \\ 4 Centre Hospitalier Régional Universitaire, Hôpital Jean Minjoz, Sérologies Parasitaires et Fongiques, \\ F-25030 Besançon, France \\ 5 Working Group Dynamyc, Faculté de Médecine, Hôpital Henri Mondor, F-94010 Créteil, France \\ * Correspondence: patrick.schwarz@med.uni-marburg.de
}

Received: 22 May 2019; Accepted: 28 June 2019; Published: 2 July 2019

\begin{abstract}
More than 20 different species of Mucorales can be responsible for human mucormycosis. Accurate identification to the species level is important. The morphological identification of Mucorales is not reliable, and the currently recommended identification standard is the molecular technique of sequencing the internal transcribed spacer regions. Nevertheless, matrix-assisted laser desorption ionization time-of-flight mass spectrometry has been shown to be an accurate alternative for the identification of bacteria, yeasts, and even filamentous fungi. Therefore, 38 Mucorales isolates, belonging to 12 different species or varieties, mainly from international collections, including 10 type or neo-type strains previously identified by molecular methods, were used to evaluate the usefulness of matrix-assisted laser desorption ionization time-of-flight mass spectrometry for the identification of human pathogenic Mucorales to the species level. One to three reference strains for each species were used to create a database of main spectrum profiles, and the remaining isolates were used as test isolates. A minimum of 10 spectra was used to build the main spectrum profile of each database strain. Interspecies discrimination for all the isolates, including species belonging to the same genus, was possible. Twenty isolates belonging to five species were used to test the database accuracy, and were correctly identified to the species level with a log-score $>2$. In summary, matrix-assisted laser desorption ionization time-of-flight mass spectrometry is a reliable and rapid method for the identification of most of the human pathogenic Mucorales to the species level.
\end{abstract}

Keywords: mucorales; mucormycosis; identification; MALDI-TOF mass spectrometry

\section{Introduction}

Mucormycosis is a life-threatening infection that is associated with a high mortality rate of about $40 \%$ [1]. The diagnosis of the disease remains difficult, and the poor outcome of the infection is related to the virulence of the fungi, and its resistance to most of the systemic antifungal drugs that are currently available [2]. An increase of the incidence of mucormycosis has been observed in the post-voriconazole era [3], and many cases of breakthrough infections in patients treated with voriconazole have been reported [4,5]. 
Mucormycosis is caused by fungi of the order Mucorales, which is a large group of fungi including very diverse species with worldwide distribution. More than 20 different species belonging to more than 10 genera can be responsible for human disease. It has been clearly shown that the different species have different antifungal susceptibility profiles [6-9]. Identification to the species level is important for better knowledge of the epidemiology of the disease and for the future optimization of the therapeutic strategies.

The identification of Mucorales to the species level remains difficult and time consuming. Indeed, identification based on classical morphology is not always reliable [10], and often, the expertise of a reference laboratory is needed. The molecular identification of Mucorales is feasible particularly by using the internal transcribed spacer regions of the ribosomal RNA as a target. In Mucorales, the internal transcribed spacer regions have low intraspecies variability and a large interspecies variability, allowing a precise identification [11-13]. Therefore, internal transcribed spacer sequencing is the gold standard for identification to the species level of isolates belonging to this group of fungi [14].

Molecular identification requires DNA extraction procedures, PCR, sequencing, and time for analyzing the results. Although most hospitals and diagnostic centers have access to a molecular laboratory, which makes this technique attractive, it is generally not used for the routine identification of all filamentous fungi isolated in clinical microbiology laboratories. In contrast, PCR has been a major advance for the diagnosis of mucormycosis, as it can be directly performed using human samples without prior cultivation of the fungus [14]. Other alternative methods of identification have also been evaluated, such as the identification based on carbohydrate assimilation with commercially available test strips [15]. Nevertheless, the technique remains less specific than internal transcribed spacer sequencing.

Mass spectroscopy using matrix-assisted laser desorption ionization time-of-flight (MALDI-TOF) has been shown to be a good alternative to molecular methods in the setting of a clinical microbiology laboratory. MALDI-TOF mass spectrometry has several important advantages over other identification methods, including simple handling, low costs, speed, and the possibility of high-throughput. MALDI-TOF mass spectrometry has been shown to be very useful for yeast identification [16]. Filamentous fungi such as Fusarium spp. as well as Aspergillus spp. [17-20] and to a lesser extent the Mucorales have also been evaluated [18,21-27].

The aim of the present study was to evaluate the accuracy of MALDI-TOF mass spectrometry for the identification of Mucorales to the species level from pure cultures.

\section{Materials and Methods}

A collection of well-characterized isolates of Mucorales has been used for this study. Isolates were obtained from international fungal collections such as CBS (Westerdijk Fungal Biodiversity Institute, Utrecht, Netherlands) and UMIP (Fungi Culture Collection of Institut Pasteur, Paris) as well as from hospital collections (microbiology laboratory of HEGP, Hôpital Européen Georges-Pompidou, and CHU Besançon, Centre Hospitalier régional Universitaire de Besançon). All the isolates were previously identified to the species level by molecular sequencing of the internal transcribed spacer regions. As far as possible, the type strain for each species was included. A total of 38 isolates from different origins (environmental, animal, and human) and from various geographical areas were included in the study.

\subsection{Extraction Protocol for MALDI-TOF Mass Spectrometry Analysis}

Sample preparation for MALDI-TOF mass spectrometry was performed as previously described with modifications [25]. The isolate was grown on solid medium until good sporulation was obtained. A mixture of spores and mycelium was harvested from the culture with the aid of a sterile cotton swab. The fungal material was transferred to a 1.5-mL microtube containing $300 \mu \mathrm{L}$ of sterile distilled water, vortexed, and $900 \mu \mathrm{L} \mathrm{70 \%} \mathrm{ethanol} \mathrm{were} \mathrm{added.} \mathrm{After} \mathrm{centrifugation} \mathrm{for} 10 \mathrm{~min}$ at 13,000 rpm, the pellet was resuspended in a mixture containing $50 \mu \mathrm{L}$ of $70 \%$ formic acid and $50 \mu \mathrm{L}$ of acetonitrile. 
After centrifugation for another $5 \mathrm{~min}$ at 13,000 rpm, $1.5 \mu \mathrm{L}$ of the supernatant was spotted onto a steel MALDI target plate (Bruker Daltonik $\mathrm{GmbH}$, Germany) and were allowed to dry at room temperature. Each spot was overlaid with $1.2 \mu \mathrm{L}$ of alpha-cyano-hydroxycinnamic acid in $2.5 \%$ of trifluoroacetic acid and $50 \%$ of acetonitrile in water (Bruker Daltonik), and air dried at room temperature. Spectra were acquired using a Microflex LT mass spectrometer (Bruker Daltonik). Spectra were recorded in positive linear mode within a mass range from 2.000 to $20.000 \mathrm{Da}$ and were analyzed using the software Flex Analysis (Bruker Daltonik).

\subsection{Evaluation of Parameters (Medium, Incubation Time)}

The influence of different parameters on the quality of spectra was evaluated. The parameters included culture medium and incubation time. Four reference strains (Lichtheimia corymbifera, Lichtheimia ramosa, Lichtheimia ornata, and Rhizopus arrhizus) were used for testing these parameters. For medium comparison, three solid media were used: malt extract agar (Fisher Scientific, Illkirch, France), RPMI 1640 agar (Biomérieux, Marcy-l'Étoile, France), and Sabouraud chloramphenicol gentamicin agar (Bio-Rad, Marnes la Coquette, France). For incubation time, two different time points were evaluated: a short incubation time of 2 days allowing profuse mycelium development and moderate sporulation, and a long incubation time of 7 days that allowed profuse sporulation. All the tests were performed at least in two independent experiments with two to four replicates for each strain.

\subsection{Construction of the Database}

The database was constructed by generating main spectrum profiles. For each species, one or two isolates (or three isolates for L. ramosa) were used to generate the main spectrum profiles. Strains were cultured on malt extract agar for 7 days at $35^{\circ} \mathrm{C}$ (except for Mucor species that were cultured at $30^{\circ} \mathrm{C}$ ). For each strain, five independent cultures were performed, and from each culture, two fungal suspensions were prepared. Extractions were performed as described above, and each sample was spotted in duplicate. Then, 20 independent spectra were generated per strain. After processing and the visual inspection of each spectrum in the software FlexAnalysis, at least 10 spectra were retained to generate the main spectrum profiles by using the software Biotyper 2.0 (Bruker Daltonik) with default parameters. The following strains $(n=18$, Table 1) were used for the construction of the database: L. corymbifera CBS 429.75, L. corymbifera CBS 100.31, L. ramosa CBS 270.65, L. ramosa CBS 269.65, L. ramosa CBS 582.65, L. ornata CBS 291.66, Mucor circinelloides f. circinelloides CBS 195.68, M. circinelloides CBS 384.95, Mucor indicus CBS 226.29, Rhizomucor miehei CBS 182.67, Rhizomucur pusillus CBS 354.68, R. pusillus IP 3.77, Rhizopus microsporus var. chinensis CBS 631.82, Rhizopus microsporus var. oligosporus CBS 112589, R. arrhizus CBS 112.07, R. oryzae IP 4.77, Syncephalastrum racemosum CBS 441.59, and Cunninghamella bertholletiae CBS 190.84.

Table 1. List of isolates used for the construction of the database.

\begin{tabular}{|c|c|c|c|}
\hline Species & Strain Number & Source & $\begin{array}{l}\text { ITS Sequence } \\
\text { Accession Number }\end{array}$ \\
\hline Rhizopus oryzae & CBS $112.07^{\mathrm{T}}$ & Human, lung & DQ119031 \\
\hline Rhizopus oryzae & IP 4.77 & Human, brain & DQ119024 \\
\hline Rhizopus microsporus var. oligosporus & CBS 112589 & Environment, tempeh & DQ119011 \\
\hline Rhizopus microsporus var. chinensis & CBS $631.72^{\mathrm{T}}$ & Environment, bread & DQ119009 \\
\hline Lichtheimia corymbifera & CBS $429.75^{\mathrm{NT}}$ & Environment, soil & FJ719407 \\
\hline Lichtheimia corymbifera & CBS 100.31 & Animal, aborted cow & FJ19398 \\
\hline Lichtheimia ramosa & CBS 269.65 & Environment, hay & FJ19405 \\
\hline Lichtheimia ramosa & CBS 270.65 & Unknown & FJ19406 \\
\hline Lichtheimia ramosa & CBS $582.65^{\mathrm{NT}}$ & Seed, Theobroma cacao & GQ342909 \\
\hline Lichtheimia ornata & CBS $291.66^{\mathrm{T}}$ & Animal, dung of bird & GQ342891 \\
\hline Mucor circinelloides f. circinelloides & CBS $195.68^{\mathrm{NT}}$ & Environment, air & DQ118991 \\
\hline Mucor circinelloides & CBS $384.95^{(\mathrm{T})}$ & Human, skin & DQ119007 \\
\hline Mucor indicus & CBS $226.29^{\mathrm{T}}$ & Unknown & DQ118994 \\
\hline Rhizomucor miehei & CBS $182.67^{\mathrm{T}}$ & Environment, plant & DQ118995 \\
\hline
\end{tabular}


Table 1. Cont.

\begin{tabular}{|c|c|c|c|}
\hline Species & Strain Number & Source & $\begin{array}{l}\text { ITS Sequence } \\
\text { Accession Number }\end{array}$ \\
\hline Rhizomucor pusillus & CBS $354.68^{\mathrm{NT}}$ & Environment, cornmeal & DQ119005 \\
\hline Rhizomucor pusillus & IP 3.77 & Animal, brain of cat & DQ119001 \\
\hline Syncephalastrum racemosum & CBS 441.59 & Animal, dung & HM999985 \\
\hline Cunninghamella bertholletiae & CBS 190.84 & Human, heart & HM849701 \\
\hline
\end{tabular}

ITS, internal transcribed spacer; IP, Institut Pasteur; CBS, Westerdijk Fungal Biodiversity Institute; T, type strain;

(T), formerly type strain of Rhizomucor variabilis var. regularior; NT, neo-type strain.

\subsection{Accuracy Test of the Database}

For the accuracy test of the database, MALDI-TOF mass spectrometry analysis was performed for a set of isolates $(n=20$, Table 2), as described above. Each isolate was tested in quadruplicate. Each spectrum was compared to the newly generated database by the MALDI Biotyper software with default parameters. The algorithm generated a log score value ranging from 0 to 3 , by comparing peak intensities, positions, and frequencies. Levels of identification were calculated and interpreted according to the manufacturer: a log score value of $>2$ indicated species identification, a log score value between $1.7-2$ indicated genus identification, and a $\log$ score value $<1.7$ indicated a non-reliable identification.

Table 2. Identification and corresponding log-score value for 20 Mucorales isolates used for the accuracy test of the database.

\begin{tabular}{llll}
\hline \multicolumn{1}{c}{ Isolate } & Collection No. & Identified Species & Score \\
\hline Rhizopus arrhizus & IP 1443.83 & Rhizopus arrhizus & 2.18 \\
Rhizopus arrhizus & CBS 120808 & Rhizopus arrhizus & 2.04 \\
Rhizopus arrhizus & CBS 120809 & Rhizopus arrhizus & 2.13 \\
Lichtheimia corymbifera & IP 1129.75 & Lichtheimia corymbifera & 2.17 \\
Lichtheimia corymbifera & IP 1279.81 & Lichtheimia corymbifera & 2.16 \\
Lichtheimia corymbifera & IP 1280.81 & Lichtheimia corymbifera & 2.09 \\
Lichtheimia corymbifera & CBS 101040 & Lichtheimia corymbifera & 2.21 \\
Lichtheimia corymbifera & CBS 120581 & Lichtheimia corymbifera & 2.04 \\
Lichtheimia corymbifera & CBS 120580 & Lichtheimia corymbifera & 2.45 \\
Lichtheimia corymbifera & BES 335 & Lichtheimia corymbifera & 2.2 \\
Lichtheimia corymbifera & PS 1.1 & Lichtheimia corymbifera & 2.07 \\
Lichtheimia ramosa & HEGP-3473 & Lichtheimia ramosa & 2.42 \\
Lichtheimia ramosa & BES 362 & Lichtheimia ramosa & 2.29 \\
Lichtheimia ramosa & BES 228 & Lichtheimia ramosa & 2.46 \\
Mucor indicus & CBS 120585 & Mucor indicus & 2.06 \\
Rhizomucor pusillus & IP 1127.75 & Rhizomucor pusillus & 2.42 \\
Rhizomucor pusillus & IP 1956.90 & Rhizomucor pusillus & 2.38 \\
Rhizomucor pusillus & CBS 120587 & Rhizomucor pusillus & 2.42 \\
Rhizomucor pusillus & CBS 120586 & Rhizomucor pusillus & 2.53 \\
Rhizomucor pusillus & CBS 120588 & Rhizomucor pusillus & 2.31 \\
\hline
\end{tabular}

IP, Institut Pasteur; CBS, Westerdijk Fungal Biodiversity Institute; BES, Centre hospitalier régional universitaire de Besançon; PS; Center for Invasive Mycoses and Antifungals Marburg; HEGP, Hôpital Européen Georges-Pompidou.

\section{Results}

\subsection{Evaluation of Parameters (Medium, Incubation Time)}

\subsubsection{Medium}

Spectra were evaluated for three Mucorales species grown on three different culture media after two different incubation times. After both time points, the spectra were similar, but the intensity of the peaks was different on the three media. For both incubation times, higher intensities of peaks were obtained on malt extract agar compared to RPMI 1640 agar and Sabouraud agar (data not shown). 
After 2 days of incubation, more peaks were obtained on RPMI 1640 agar, whereas at day 7, more peaks were obtained on Sabouraud agar. Nevertheless, the spectra remained similar on the three media, and differences were species-dependent (data not shown). Globally, malt extract agar seemed to be the most reliable medium, and was chosen for the subsequent experiments.

\subsubsection{Incubation time}

Two incubation times ( 2 days and 7 days) were further analyzed on malt extract agar. Overall, there were some differences in the presence and intensities of peaks. To ensure good identification of all the species, and as slow-growing species sporulate insufficiently after 2 days of growth, 7 days of incubation were chosen as the endpoint for the database construction.

\subsection{Construction of the Database}

After these preliminary experiments that allowed the determination of the medium and incubation time, the database was set up. For assessing the intergenera, interspecies, and intraspecies variability, all the main spectrum profiles from the database were identified against the database itself. The results of the log-score values are presented in Table 3. A log-score of 3 indicated a perfect match (i.e., when a database strain was compared to itself). A high intergenera variability was observed with a log-score $<1.1$ for all the strains. The highest similarity between two isolates belonging to different genera was obtained between M. indicus and R. pusillus (log-score value of 1.01). Similarly, high interspecies variabilities were found for all the species belonging to the Lichtheimia (highest log-score of 1.14 between L. ramosa and L. ornata), Mucor, Rhizomucor, and Rhizopus genus. Examples of spectra of several species are presented in Figure 1, showing the specific patterns for each species. Within a given species, similarity was generally high (log-score up to 2.49 for strains of L. corymbifera and 2.82 of R. pusillus), but some intraspecies variability was also observed for L. ramosa, M. circinelloides, R. microsporus, and R. arrhizus.

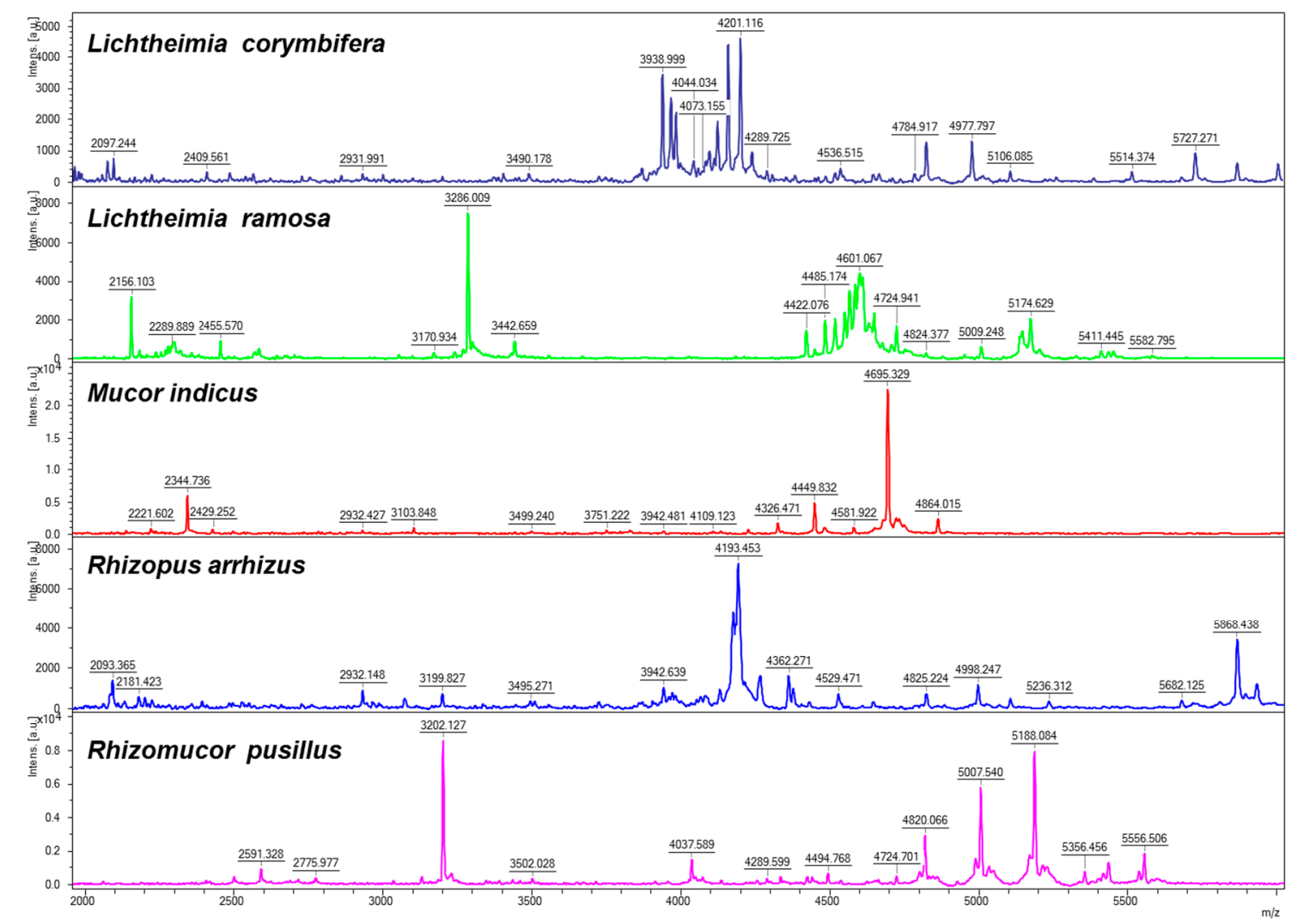

Figure 1. Typical spectra patterns obtained for five different species of Mucorales. 
Table 3. Log-score values obtained when the main spectrum profiles were identified by the database itself. For each isolate, the log-score values for the first 10 best matches are shown.

\begin{tabular}{|c|c|c|c|c|c|c|c|c|c|c|c|c|c|c|c|c|c|c|}
\hline \multirow{2}{*}{ Isolate Number and Identification } & \multicolumn{18}{|c|}{ Log-Score Values for Strain } \\
\hline & 1 & 2 & 3 & 4 & 5 & 6 & 7 & 8 & 9 & 10 & 11 & 12 & 13 & 14 & 15 & 16 & 17 & 18 \\
\hline 1- C. bertholletiae CBS 190.84 & 3 & 0.57 & 0.42 & ND & 0.43 & 0.38 & ND & 0.38 & ND & ND & ND & 0.52 & 0.35 & ND & ND & ND & 0.28 & 0.58 \\
\hline 2- L. corymbifera CBS 100.31 & 0.47 & 3 & 2.39 & 0.85 & ND & 0.18 & 0.66 & 0.6 & ND & 0.39 & ND & ND & ND & 0.23 & 0.35 & ND & ND & ND \\
\hline 4- L. ornata CBS 291.66 & ND & 0.8 & 1.13 & 3 & 0.35 & 0.67 & 0.67 & ND & ND & 0.91 & ND & ND & ND & 0.54 & ND & 0.4 & 0.38 & ND \\
\hline 5- L. ramosa CBS 269.65 & 0.39 & ND & ND & 0.58 & 3 & 1.78 & 1.04 & 0.44 & 0.21 & 0.58 & ND & ND & ND & 0.51 & ND & ND & 0.37 & ND \\
\hline 6- L. ramosa CBS 270.35 & 0.41 & ND & 0.58 & 0.6 & 1.76 & 3 & 0.87 & ND & ND & ND & 0.52 & ND & ND & 0.4 & 0.47 & ND & 0.44 & ND \\
\hline 7- L. ramosa CBS 582.65 & ND & 0.42 & ND & 0.65 & 1.07 & 0.98 & 3 & ND & ND & 0.27 & ND & 0.7 & 0.34 & 0.38 & 0.32 & ND & ND & ND \\
\hline 8- M. circinelloides CBS 195.68 & 0.58 & ND & ND & ND & 0.44 & 0.29 & ND & 3 & 0.87 & 0.98 & ND & ND & ND & 0.9 & 0.75 & 0.28 & ND & ND \\
\hline 9- M. circinelloides CBS 384.95 & 0.14 & ND & ND & 0.1 & 0.1 & ND & ND & 0.9 & 3 & 0.63 & ND & ND & ND & 0.76 & 0.25 & ND & 0.31 & 0.13 \\
\hline 10- M. indicus CBS 226.29 & ND & ND & ND & 0.95 & 0.55 & 0.66 & ND & 0.98 & 0.62 & 3 & ND & 0.59 & 0.98 & 0.53 & ND & 0.9 & ND & ND \\
\hline 11- R. miehei CBS 182.67 & 0.23 & 0.16 & 0.04 & ND & ND & 0.54 & ND & ND & ND & 0.19 & 3 & 0.05 & 0.27 & 0.23 & ND & ND & ND & 0.22 \\
\hline 12- R. pusillus CBS 354.68 & 0.48 & ND & ND & ND & ND & 0.27 & 0.71 & ND & ND & 0.57 & 0.09 & 3 & 2.82 & 0.38 & 0.64 & 0.31 & ND & ND \\
\hline 13- R. pusillus IP 3.77 & 0.3 & ND & 0.33 & ND & ND & ND & 0.35 & ND & ND & 1.01 & 0.29 & 2.82 & 3 & 0.33 & 0.47 & 0.04 & ND & ND \\
\hline 14- R. microsporus CBS 631.82 & ND & ND & ND & 0.61 & 0.53 & 0.38 & ND & 0.94 & 0.78 & 0.57 & ND & 0.38 & ND & 3 & 1.54 & ND & 0.43 & ND \\
\hline 15- R. microsporus IP 1126.75 & ND & ND & ND & ND & ND & 0.47 & 0.31 & 0.81 & 0.24 & 0.42 & ND & 0.66 & 0.5 & 1.54 & 3 & ND & ND & 0.3 \\
\hline 16- R. arrhizus CBS 112.07 & ND & ND & ND & 0.41 & ND & 0.66 & ND & 0.31 & ND & 0.92 & 0.05 & 0.21 & ND & ND & ND & 3 & 0.47 & 0.58 \\
\hline 17- R. arrhizus IP 4.77 & 0.33 & ND & ND & 0.42 & 0.5 & 0.45 & 0.25 & ND & 0.3 & ND & ND & ND & ND & 0.45 & 0.11 & 0.41 & 3 & ND \\
\hline 18- S. racemosum CBS 441.59 & 0.62 & ND & ND & ND & 0.16 & 0.3 & ND & ND & 0.14 & 0.3 & 0.27 & ND & ND & ND & 0.25 & 0.81 & 0.46 & 3 \\
\hline
\end{tabular}

IP, Institut Pasteur; CBS, Westerdijk Fungal Biodiversity Institute; ND, not determined. 


\subsection{Accuracy Test of the Database}

Twenty different isolates belonging to the major species (and not used for the construction of the database) were spotted in quadruplicate, and identification was performed using the newly generated database by calculation of the log-score values. Isolates belonged to the most common pathogenic species, namely R. arrhizus, L. corymbifera, L. ramosa, Mucor indicus, and R. pusillus. The log-score values obtained for all the isolates tested are shown in Table 2. For all the tested isolates, high log-score values of $>2$ were obtained. For each of the strains, a more detailed analysis was also performed. The 10 best matches were recorded, and log-score values obtained for strains belonging to the same species (measuring the intraspecies variability) and different species (measuring the interspecies variability) were analyzed (Figure 2). For L. corymbifera and R. pusillus, no overlap of log-score values was seen, indicating that all the tested isolates of these species exhibit a high similarity. In contrast, for $L$. ramosa and $R$. arrhizus, log-score values of $<2$ were obtained. This indicates that the isolates of these species show a certain degree of interspecies variability. Nevertheless, this did not preclude the correct identification to the species level (Table 2).

Rhizopus arrhizus

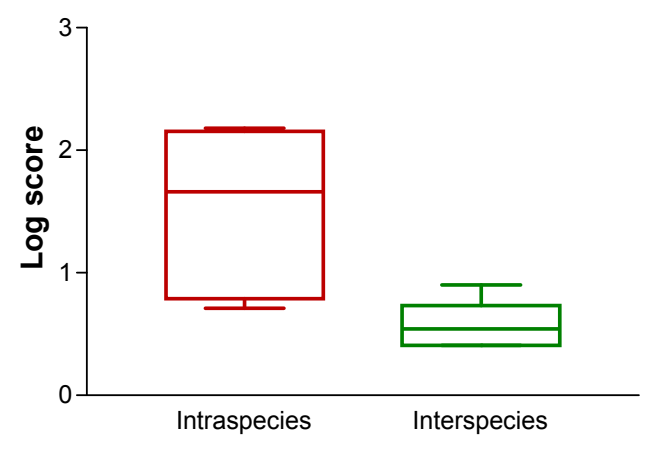

Lichtheimia ramosa

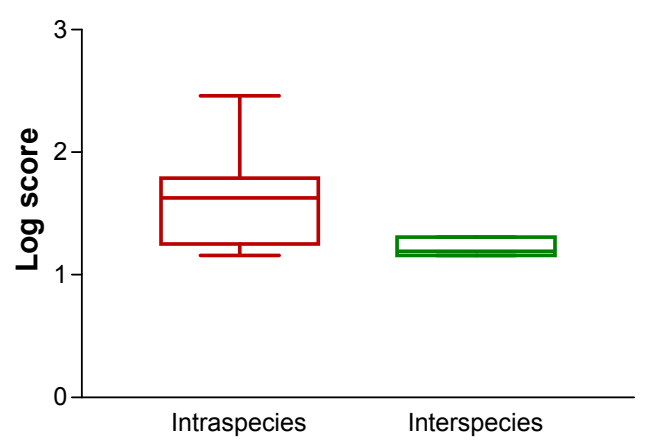

Lichtheimia corymbifera

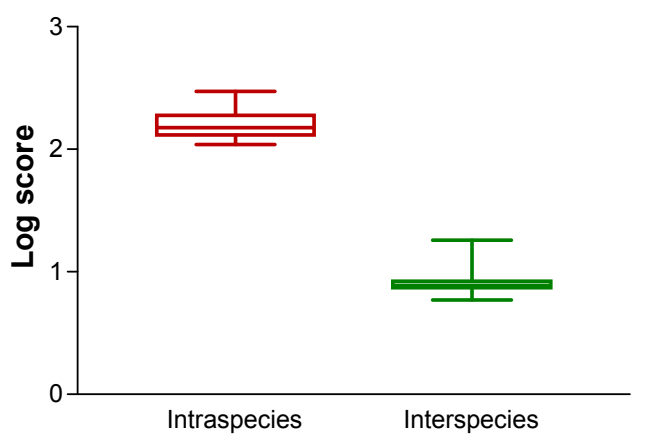

Rhizomucor pusillus

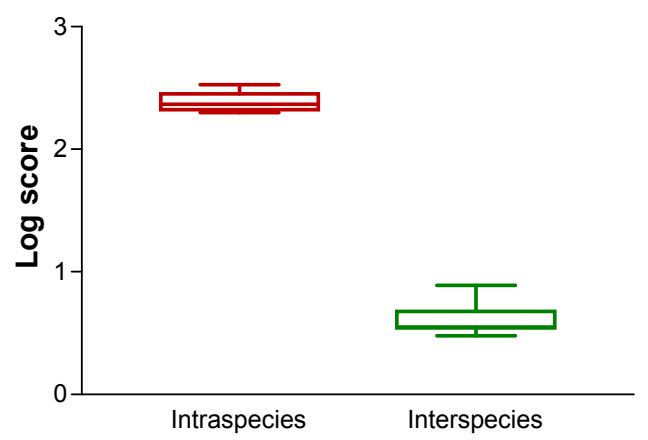

Figure 2. Plot of log-score values obtained for the isolates, belonging to four species, were used for the accuracy test of the database. On each graph, the left panel plots the log-scores values obtained for isolates belonging to the same species (as a marker of intraspecies variability), and the right panel plots the log-scores values obtained for isolates belonging to different species (as a marker of interspecies variability).

\section{Discussion}

MALDI-TOF mass spectrometry is widely used for the identification of bacteria [28] and yeasts [16] to the species level. Major advantages of this identification technique compared to molecular-based identification are its accuracy combined with simplicity, cost effectiveness, and the possibility of high 
throughput. Nevertheless, the technique also has disadvantages, as the isolates have to be grown as pure cultures, and identification directly from clinical specimens is not possible. Additionally, the identification of filamentous fungi remains challenging, as differences in incubation time, medium selection, and sample preparation may influence the results [29]. Moreover, commercial libraries are incomplete, and often lack a significant number of species and isolates [30]. Therefore, we set up a standardized technique for the sample preparation, and built our own database for the identification of Mucorales to the species level, including reference strains for the most common species responsible for human disease.

Our results demonstrate that a precise discrimination of Mucorales species with a high variability between genera, and also between species, is possible. Intraspecies variability was species-dependent: for some species, a low variability was seen, while for R. arrhizus and L. ramosa, a certain degree of intraspecies variability was obtained. One limitation of this study is that for some species, only a relatively low number of isolates was included. That might be the reason why for those species, no intraspecies variability was observed. The intraspecies variability of $R$. arrhizus $[18,21,22,24,26,27]$ and L. ramosa $[25,27]$ was already reported by others. Our results are in accordance with these findings. Taking these findings into account, it is of major importance to include several isolates of each species into the database to maximize the possibility of reliable identification.

The accuracy of identification of Mucorales to the species level by MALDI-TOF mass spectrometry has already been evaluated by other studies. In one study, a database was set up, including a total of 19 isolates belonging to Mucor, Lichtheimia, Rhizopus spp., and R. pusillus. The database was further tested for accuracy with eight Mucorales isolates, including one R. arrhizus and four L. ramosa strains. No problems in identification were reported; all the log score values were $>2$ [23]. Another study analyzed the identification of pathogenic Lichtheimia species [25]. The database comprised 12 Lichtheimia strains belonging to five different species, and seven strains belonging to seven different Mucorales species were also added. The database was tested with 34 additional Lichtheimia isolates. All the isolates of L. corymbifera, L. ornata, Lichtheimia hyalospora, and Lichtheimia sphaerocystis were correctly identified when taking the highest obtained log-score value into account. When compared to other species, log-score values $>2$ were obtained, indicating a false identification. The identification of L. ramosa strains was even more difficult; log-score values of $<1.9$ were obtained for two of the 17 tested isolates. Aggravatingly, some isolates exhibited the highest obtained log-score values for a different species, indicating a false identification [25]. Despite the intraspecies variability of L. ramosa, in our database, no false identifications were seen for the Lichtheimia species. Problems in the identification of $L$. ramosa isolates were also seen in another study evaluating the commercialized Bruker library alone or in combination with an in-house library [27]. The databases were tested with 111 isolates. Despite a significant increase from $49.5 \%$ to $81.1 \%$ of isolates identified correctly to the species level, when using both libraries compared to the Bruker library alone, seven isolates, including one L. ornata and three L. ramosa isolates were misidentified. For the other three tested isolates of L. ramosa, only an identification to the genus level was possible (log-score value $\geq 1.7$ ). The intraspecies variability of L. ramosa may be related to the existence of at least two different clades within this species, as described previously [31]. In the same study, the identification of $R$. arrhizus isolates was less problematic. Only one of 20 isolates was not identified to the species level [27]. In our study, all three R. arrhizus isolates were correctly identified with log-score values $>2$. Analysis of the spectra of $R$. arrhizus isolates revealed two different spectrum types with different peaks and different peak intensities [22]. Two different types of $R$. arrhizus isolates were also found in another study evaluating the spectra of 14 R. arrhizus isolates. Interestingly, the spectra of five R. arrhizus isolates were more closely related to 15 Rhizopus delemar isolates than to the remaining nine R. arrhizus isolates [24]. In a study, evaluating a huge in-house library with 472 filamentous fungal species, including Mucorales, combined with the Burker library, also encountered problems in the identification of $R$. arrhizus isolates. Identification was only possible to the genus level (log-score values $>1.7$ and $<2$ ) [21]. The National Institutes of Health also designed an in-house library containing 152 different mold species, including 27 Mucorales isolates. 
While no problems were reported when challenged by three R. arrhizus isolates, the identification of the same isolates by the Burker database was impossible. Identification of all the other tested Mucorales isolates $(n=9)$ by the National Institutes of Health database was successful [18]. Problems in the identification of Mucorales to the species level using the Burker database were also reported by others [26]. Globally, different results for the identification of Mucorales to the species level by MALDI-TOF mass spectrometry were obtained, especially for those species showing intraspecies variability. Nevertheless, as long as the database used for identification comprises a sufficient number of reference isolates for each species, identification to the species level seems to be possible.

\section{Conclusion}

We were able to demonstrate that MALDI-TOF mass spectrometry, which is rapid, and cost-effective, is a reliable method for the identification of Mucorales to the species level. It could be useful to implement the database with more clinical and environmental isolates belonging to the different species for even better accuracy.

Author Contributions: Conceptualization and study design, E.D. and F.G.; experiments and data acquisition, H.G. and F.L.; data analysis, H.G., F.L., P.S, and E.D.; writing-original draft of the manuscript, P.S.; writing-review and editing, all authors.

Funding: This research was funded by an educational grant from Gilead Sciences, grant number IX-FR-131-0288.

Conflicts of Interest: P.S. has received research grants from Basilea Pharmaceutica, Gilead and Pfizer, and has received travel grants from Gilead and Pfizer. During the past 5years, E.D. has received: research grants from MSD and Gilead; travel grants from Gilead, MSD, Pfizer and Astellas; and speaker's fees from Gilead, MSD and Astellas. H.G., F.L. and F.G.: none to declare.

\section{References}

1. Roden, M.M.; Zaoutis, T.E.; Buchanan, W.L.; Knudsen, T.A.; Sarkisova, T.A.; Schaufele, R.L.; Sein, M.; Sein, T.; Chiou, C.C.; Chu, J.H.; et al. Epidemiology and outcome of zygomycosis: A review of 929 reported cases. Clin. Infect. Dis. 2005, 41, 634-653. [CrossRef]

2. Dannaoui, E.; Garcia-Hermoso, D. The Zygomycetes. In New Insights in Fungal Pathogenity; Kavanagh, K., Ed.; Springer Science: Dordrecht, The Netherlands, 2007; pp. 159-183.

3. Bitar, D.; van Cauteren, D.; Lanternier, F.; Dannaoui, E.; Che, D.; Dromer, F.; Desenclos, J.C.; Lortholary, O. Increasing incidence of zygomycosis (mucormycosis), France, 1997-2006. Emerg. Infect. Dis. 2009, 15, 1395-1401. [CrossRef]

4. Lamoth, F.; Chung, S.J.; Damonti, L.; Alexander, B.D. Changing epidemiology of invasive mold infections in patients receiving azole prophylaxis. Clin. Infect. Dis. 2017, 64, 1619-1621. [CrossRef]

5. Marty, F.M.; Cosimi, L.A.; Baden, L.R. Breakthrough zygomycosis after voriconazole treatment in recipients of hematopoietic stem-cell transplants. N. Engl. J. Med. 2004, 350, 950-952. [CrossRef]

6. Alastruey-Izquierdo, A.; Castelli, M.V.; Cuesta, I.; Zaragoza, O.; Monzon, A.; Mellado, E.; Rodriguez-Tudela, J.L. In vitro activity of antifungals against Zygomycetes. Clin. Microbiol. Infect. 2009, 15 (Suppl. 5), 71-76. [CrossRef]

7. Dannaoui, E. Antifungal resistance in Mucorales. Int. J. Antimicrob. Agents 2017, 50, 617-621. [CrossRef]

8. Dannaoui, E.; Meletiadis, J.; Mouton, J.W.; Meis, J.F.; Verweij, P.E. In vitro susceptibilities of zygomycetes to conventional and new antifungals. J. Antimicrob. Chemother. 2003, 51, 45-52. [CrossRef]

9. Vitale, R.G.; de Hoog, G.S.; Schwarz, P.; Dannaoui, E.; Deng, S.; Machouart, M.; Voigt, K.; van de Sande, W.W.; Dolatabadi, S.; Meis, J.F.; et al. Antifungal susceptibility and phylogeny of opportunistic members of the order Mucorales. J. Clin. Microbiol. 2012, 50, 66-75. [CrossRef]

10. Kontoyiannis, D.P.; Lionakis, M.S.; Lewis, R.E.; Chamilos, G.; Healy, M.; Perego, C.; Safdar, A.; Kantarjian, H.; Champlin, R.; Walsh, T.J.; et al. Zygomycosis in a tertiary-care cancer center in the era of Aspergillus-active antifungal therapy: A case-control observational study of 27 recent cases. J. Infect. Dis. 2005, 191, 1350-1360. [CrossRef]

11. CLSI. Interpretive Criteria for Identification of Bacteria and Fungi by Targeted DNA Sequencing; Approved Guideline MM18; Clinical and Laboratory Standards Institute: Alexandria, VA, USA, 2018. 
12. Balajee, S.A.; Borman, A.M.; Brandt, M.E.; Cano, J.; Cuenca-Estrella, M.; Dannaoui, E.; Guarro, J.; Haase, G.; Kibbler, C.C.; Meyer, W.; et al. Wickes, Sequence-based identification of Aspergillus, Fusarium, and Mucorales species in the clinical mycology laboratory: Where are we and where should we go from here? J. Clin. Microbiol. 2009, 47, 877-884. [CrossRef]

13. Schwarz, P.; Bretagne, S.; Gantier, J.C.; Garcia-Hermoso, D.; Lortholary, O.; Dromer, F.; Dannaoui, E. Molecular identification of zygomycetes from culture and experimentally infected tissues. J. Clin. Microbiol. 2006, 44, 340-349. [CrossRef]

14. Cornely, O.A.; Arikan-Akdagli, S.; Dannaoui, E.; Groll, A.H.; Lagrou, K.; Chakrabarti, A.; Lanternier, F.; Pagano, L.; Skiada, A.; Akova, M.; et al. ESCMID and ECMM joint clinical guidelines for the diagnosis and management of mucormycosis 2013. Clin. Microbiol. Infect. 2014, 20 (Suppl. 3), 5-26. [CrossRef]

15. Schwarz, P.; Lortholary, O.; Dromer, F.; Dannaoui, E. Carbon assimilation profiles as a tool for identification of zygomycetes. J. Clin. Microbiol. 2007, 45, 1433-1439. [CrossRef]

16. Buchan, B.W.; Ledeboer, N.A. Advances in identification of clinical yeast isolates by use of matrix-assisted laser desorption ionization-time of flight mass spectrometry. J. Clin. Microbiol. 2013, 51, 1359-1366. [CrossRef]

17. Cassagne, C.; Normand, A.C.; L'Ollivier, C.; Ranque, S.; Piarroux, R. Performance of MALDI-TOF MS platforms for fungal identification. Mycoses 2016, 59, 678-690. [CrossRef]

18. Lau, A.F.; Drake, S.K.; Calhoun, L.B.; Henderson, C.M.; Zelazny, A.M. Development of a clinically comprehensive database and a simple procedure for identification of molds from solid media by matrix-assisted laser desorption ionization-time of flight mass spectrometry. J. Clin. Microbiol. 2013, 51, 828-834. [CrossRef]

19. Marinach-Patrice, C.; Lethuillier, A.; Marly, A.; Brossas, J.Y.; Gene, J.; Symoens, F.; Datry, A.; Guarro, J.; Mazier, D.; Hennequin, C. Use of mass spectrometry to identify clinical Fusarium isolates. Clin. Microbiol. Infect. 2009, 15, 634-642. [CrossRef]

20. Sanguinetti, M.; Posteraro, B. Identification of molds by matrix-assisted laser desorption ionization-time of flight mass spectrometry. J. Clin. Microbiol. 2017, 55, 369-379. [CrossRef]

21. Becker, P.T.; De Bel, A.; Martiny, D.; Ranque, S.; Piarroux, R.; Cassagne, C.; Detandt, M.; Hendrickx, M. Identification of filamentous fungi isolates by MALDI-TOF mass spectrometry: Clinical evaluation of an extended reference spectra library. Med. Mycol. 2014, 52, 826-834. [CrossRef]

22. Chen, Y.S.; Liu, Y.H.; Teng, S.H.; Liao, C.H.; Hung, C.C.; Sheng, W.H.; Teng, L.J.; Hsueh, P.R. Evaluation of the matrix-assisted laser desorption/ionization time-of-flight mass spectrometry Bruker Biotyper for identification of Penicillium marneffei, Paecilomyces species, Fusarium solani, Rhizopus species, and Pseudallescheria boydii. Front. Microbiol. 2015, 6, 679. [CrossRef]

23. De Carolis, E.; Posteraro, B.; Lass-Florl, C.; Vella, A.; Florio, A.R.; Torelli, R.; Girmenia, C.; Colozza, C.; Tortorano, A.M.; Sanguinetti, M.; et al. Species identification of Aspergillus, Fusarium and Mucorales with direct surface analysis by matrix-assisted laser desorption ionization time-of-flight mass spectrometry. Clin. Microbiol. Infect. 2012, 18, 475-484. [CrossRef]

24. Dolatabadi, S.; Kolecka, A.; Versteeg, M.; de Hoog, S.G.; Boekhout, T. Differentiation of clinically relevant Mucorales Rhizopus microsporus and R. arrhizus by matrix-assisted laser desorption ionization time-of-flight mass spectrometry (MALDI-TOF MS). J. Med. Microbiol. 2015, 64, 694-701.

25. Schrödl, W.; Heydel, T.; Schwartze, V.U.; Hoffmann, K.; Grosse-Herrenthey, A.; Walther, G.; Alastruey-Izquierdo, A.; Rodriguez-Tudela, J.L.; Olias, P.; Jacobsen, I.D.; et al. Direct analysis and identification of pathogenic Lichtheimia species by matrix-assisted laser desorption ionization-time of flight analyzer-mediated mass spectrometry. J. Clin. Microbiol. 2012, 50, 419-427. [CrossRef]

26. Schulthess, B.; Ledermann, R.; Mouttet, F.; Zbinden, A.; Bloemberg, G.V.; Bottger, E.C.; Hombach, M. Use of the Bruker MALDI Biotyper for identification of molds in the clinical mycology laboratory. J. Clin. Microbiol. 2014, 52, 2797-2803. [CrossRef]

27. Shao, J.; Wan, Z.; Li, R.; Yu, J. Species identification and delineation of pathogenic Mucorales by matrix-assisted laser desorption ionization-time of flight mass spectrometry. J. Clin. Microbiol. 2018, 56, e01886-17. [CrossRef]

28. Jamal, W.; Albert, M.J.; Rotimi, V.O. Real-time comparative evaluation of bioMerieux VITEK MS versus Bruker Microflex MS, two matrix-assisted laser desorption-ionization time-of-flight mass spectrometry systems, for identification of clinically significant bacteria. BMC Microbiol. 2014, 14, 289. [CrossRef] 
29. Packeu, A.; de Bel, A.; l'Ollivier, C.; Ranque, S.; Detandt, M.; Hendrickx, M. Fast and accurate identification of dermatophytes by matrix-assisted laser desorption ionization-time of flight mass spectrometry: Validation in the clinical laboratory. J. Clin. Microbiol. 2014, 52, 3440-3443. [CrossRef]

30. Sleiman, S.; Halliday, C.L.; Chapman, B.; Brown, M.; Nitschke, J.; Lau, A.F.; Chen, S.C. Performance of matrix-assisted laser desorption ionization-time of flight sass spectrometry for identification of Aspergillus, Scedosporium, and Fusarium spp. in the Australian clinical setting. J. Clin. Microbiol. 2016, 54, 2182-2186. [CrossRef]

31. Alastruey-Izquierdo, A.; Hoffmann, K.; de Hoog, G.S.; Rodriguez-Tudela, J.L.; Voigt, K.; Bibashi, E.; Walther, G. Species recognition and clinical relevance of the zygomycetous genus Lichtheimia (syn. Absidia pro parte, Mycocladus). J. Clin. Microbiol. 2010, 48, 2154-2170.

(C) 2019 by the authors. Licensee MDPI, Basel, Switzerland. This article is an open access article distributed under the terms and conditions of the Creative Commons Attribution (CC BY) license (http://creativecommons.org/licenses/by/4.0/). 